\title{
Daily vision testing can expose the prodromal phase of migraine.
}

Allison M. McKendrick ${ }^{1}$, Yu Man Chan ${ }^{1}$, Algis J Vingrys ${ }^{1}$, Andrew Turpin ${ }^{2}$, David R Badcock ${ }^{3}$

${ }^{1}$ University of Melbourne, Department of Optometry and Vision Sciences, Melbourne, Australia

${ }^{2}$ University of Melbourne, Department of Computing and Information System, Melbourne, Australia

${ }^{3}$ University of Western Australia, School of Psychological Science, Perth, Australia

Funding: Project supported by Australian National Health and Medical Research Council Project Grant APPID1081874 


\begin{abstract}
Background: Several visual tasks have been proposed as indirect assays of the balance between cortical inhibition and excitation in migraine. This study aimed to determine whether daily measurement of performance on such tasks can reveal perceptual changes in the build up to migraine events.
\end{abstract}

Methods: Visual performance was measured daily at home in 16 non-headache controls and 18 individuals with migraine using a testing protocol on a portable tablet device. Observers performed two tasks: luminance increment detection in spatial luminance noise and centre surround contrast suppression.

Results Luminance thresholds were reduced in migraine compared to control groups $(\mathrm{p}<0.05)$, but thresholds did not alter across the migraine cycle. While headache-free, centre-surround contrast suppression was stronger for the migraine group relative to controls $(\mathrm{p}<0.05)$. Surround suppression weakened at around 48 hours prior to a migraine attack and strengthened to approach their headache-free by 24 hours post-migraine (main effect of timing, $\mathrm{p}<0.05$ ).

Conclusions: Daily portable testing of vision enabled insight to perceptual performance in the lead up to migraine events, a time point that is typically difficult to capture experimentally. Perceptual surround suppression of contrast fluctuates during the migraine cycle, supporting the utility of this measure as an indirect, non-invasive assay of the balance between cortical inhibition and excitation. 


\section{Introduction}

Migraine is a cyclic disorder including prodromal stages, attack, and recovery (1). Functional assessment of migraine patients at different stages of this cycle may contribute to a more comprehensive understanding of migraine, however there are logistical challenges involved, particularly in capturing the days immediately prior to a migraine. Techniques such as neuroimaging and electrophysiology are extremely useful for studying migraine pathophysiology (2-6), however they are costly and require specialised equipment. Because these tests are typically repeated at sporadic intervals within individuals, it is highly challenging to define functional changes arising during the time course of a migraine event precisely with such methods. An alternate, feasible, approach to tracking may be to use a perceptual test on a tablet device that allows portable daily testing.

While specific triggers, and functional biomarkers, for various stages in the migraine cycle are not fully understood, convergent evidence from neuroimaging, electrophysiology, and visual processing points towards fluctuations in the balance between cortical inhibition and excitation across days (2, 6-12). Visual perceptual tasks that depend, at least in part, on the balance of visual cortical inhibition versus excitation have the potential to be useful indicators of impending migraine onset when used for daily assessment, if indeed their results vary systematically with migraine status and onset. One candidate task is the Chubb illusion (13), whereby the perceived contrast of a grating patch is reduced in the presence of a high contrast surround, due to inhibitory lateral connections and 4 
extrastriate feedback effects on the central excitatory zone (centre-surround contrast suppression) (14-18). Interictally, people who experience migraine typically perceive the central grating at lower contrast (more suppressed) than non-headache controls (19). The precise mechanisms underpinning this effect are unclear; with such spatial contrast inhibition driven both directly by lateral inhibitory interneurons, in addition to excitatory extrastriate feedback to lateral inhibitory interneurons (14-18). Such centre-surround contrast tasks have been used to indirectly measure the balance between inhibition and excitation in a range of clinical conditions, including schizophrenia (20, 21), healthy aging (22) and epilepsy (23). In the context of migraine, a key missing piece of information is whether the strength of the Chubb illusion fluctuates predictably during the migraine cycle.

Deficits in the ability to detect luminance increments have been well studied in migraine, typically using perimetry to assess across the visual field, and have been shown to vary in severity with duration post-migraine $(24,25)$. We chose our second visual task to be a variant of luminance increment detection, where the increments were superimposed on spatial luminance noise $(26,27)$. Previous studies have demonstrated interictal elevation of threshold in migraine groups suggestive of an imbalance in cortical excitation and inhibition that leads to excessive neural noise $(26,27)$. Neural noise can arise from random neural firing in the absence of a stimulus (additive noise) or due to excessive firing in response to a stimulus (multiplicative noise). By testing at high, medium and low spatial noise conditions, Webster and colleagues (2012) revealed alterations that were interpreted by the authors as possibly arising from differences in multiplicative neural noise in their migraine group. 
Participants were tested interictally, when entirely symptom-free. A key unanswered question is whether performance on this task fluctuates in the build up to a migraine event.

In this study, we monitor visual performance daily using an at-home testing protocol on a portable tablet device, and compare individuals with migraine at various times in their migraine cycle, to the daily fluctuation of performance in non-headache controls. Specifically, we measured the strength of centre surround contrast suppression (Chubb illusion), in addition to luminance increment detection in spatial luminance noise. Assuming that cortical excitation increases in the lead up to a migraine and then relatively normalises post-migraine, the following patterns of performance are hypothesised: 1) contrast suppression should decrease immediately pre-migraine and increase post migraine; 2) luminance increment thresholds should increase pre-migraine and decrease post migraine. Consequently, the aim of this study was to identify if there was any trend in the daily perceptual measures across days pre- and post-migraine, specifically to test the predictions described above.

\section{Methods}

\section{General experimental outline}

Participants attended twice, once before and once after the at-home testing phase. At the initial session, observers were trained to use the tablet and perform the tasks. Subsequently, participants were asked to complete all parts of the daily testing without substantive breaks, at a time of day when 
they anticipated minimal distraction, and at approximately the same time each day. Control participants performed the daily tasks over a 14-day period. People with migraine conducted daily testing until they experienced a migraine event, and then for the following week. As part of the daily testing, migraine participants were required to indicate via a survey question embedded in the tablet app if they had experienced a migraine in the past 24 hours. If they answered "yes”, the participant was contacted via telephone within the next 48 hours to confirm that the event was consistent with a typical migraine with or without aura. In an attempt to improve compliance, all participants received a thank you message (via their indicated preferred mode of contact: text message on their mobile device or email) every 3 to 7 days during the home monitoring phase. If they missed a day of testing, participants were contacted via voice call or text message on their preferred mode of contact (mobile number or email), to check if they had forgotten or had any technical issues with the tablet. They were asked to resume daily testing as soon as possible. All participants returned for a final laboratory session where they performed the tasks on the tablet once more under supervision (author YMC) to allow comparison between the performance measured in the initial (Lab 1) and final (Lab 2) sessions to quantify any practice effects arising from the daily home testing period. All migraine participants were headache-free and asymptomatic at the two in-office sessions. The home testing was completed in no more than 10 minutes each day. Details of the specific tests appear below. 


\section{Participants}

Data was collected and analysed for sixteen non-headache controls (mean age 26.8, 19-46 years, 5 males), 10 people with migraine with aura (MA) (mean age 32.1, 19-47 years, 2 males) and 8 people with migraine without aura (MO) (mean age 33.1, 20-49 years, 1 male). An additional 3 individuals with migraine enrolled in the study but were excluded after they did not have a migraine event within 2 months of home testing. A minimum sample size of 15 controls and 15 with migraine was estimated to find between group differences during the interictal period (19). Participants were recruited from our laboratory database and advertisements placed within the university during the period August 2015 to April 2017. Migraine participants had been diagnosed with migraine by their general practitioner or neurologist and had symptoms consistent with the International Classification of Headache Disorders (ICHD)-II criteria for migraine with (MA) or without (MO) aura (28). A clinical migraine history was recorded including the number of days since their last migraine (median; interquartile range (IQR): 7, 2-30 days), number of attacks per year (median, IQR: 19, 1528 attacks) and symptoms required to confirm MO or MA. Impact of migraine was graded using the Migraine Disability Assessment (MIDAS) (median, IQR: 3, 1-4). Participants were required to experience migraines every 1-4 weeks to ensure that a headache-free period and at least one migraine attack could be captured within a reasonable duration of the home testing (ideally within a month). Those with chronic migraine were excluded. 
Control participants had never experienced a migraine, or migraine aura. In addition, non-headache status was defined as no more than four headaches per year, with those headache events clearly explicable (for example, influenza or dehydration). All participants underwent a brief optometric screening to ensure normal vision. All had refractive errors within $\pm 5 \mathrm{D}$ spherical and less than $2 \mathrm{D}$ cylindrical, with best corrected visual acuity of 6/7.5 or better, and normal ophthalmic health. Participants were not permitted to suffer from any other neurological conditions, nor to take regular medications known to affect vision or cognition, including migraine prophylactics. Project ethics approval was provided by the Human Research Ethics Committee of the University of Melbourne (UMHREC 1443362.2) and all participants provided written informed consent in accordance with protocols defined in the Declaration of Helsinki.

\section{Equipment}

Visual tasks were conducted using the open access application PsyPad 2.0 (29) on iPad 2 devices (1024×768 pixel, 256 bits per pixel, $30 \mathrm{~Hz}$, max luminance $344.5 \mathrm{~cd} / \mathrm{m}^{2}$; Apple, California, USA). Observers were loaned an iPad and provided with a tape measure to perform tasks binocularly from $40 \mathrm{~cm}$ viewing distance. The iPads did not have other apps installed. Each iPad was set to turn "autobrightness" off and to set screen brightness to maximum. Participants received written instructions on how to check that auto-brightness was off prior to testing, and also how to check that screen 9 
brightness was set to maximum. Participants were also shown how to check these settings during the initial visit, and compliance with this behaviour was verbally queried during the final follow-up visit.

\section{Stimuli}

Figure $1 \mathrm{~A}$ and $1 \mathrm{~B}$ represent the luminance increment detection stimulus in high and low spatial luminance noise respectively. Two spatial luminance noise squares (each 4 deg of visual angle) were presented with centres 5.5 deg from the screen centre, on a mean background luminance of 201 $\mathrm{cd} / \mathrm{m}^{2}$. Noise squares were filled with noise super-pixels, each 6 × 6 standard pixel $(0.2 \times 0.2$ deg of visual angle). The luminance noise was randomly sampled from a Gaussian distribution (mean = 0; stdev $=26.25 \mathrm{~cd} / \mathrm{m}^{2}$ (low noise) or $52.5 \mathrm{~cd} / \mathrm{m}^{2}$ (high noise)). One of the squares was randomly selected to present a superimposed luminance increment (1.5 deg diameter circle). Stimuli duration was 500 ms. Observers were instructed to make a quick eye movement to view both squares before indicating whether the luminance increment appeared on the left or right. Observers indicated their response by tapping on touchscreen regions (3.4 x 3.4 deg of visual angle) at the bottom left and right corners of the display. The next stimulus was presented $500 \mathrm{~ms}$ after a response. The luminance of the circle varied according to two interleaved 3-down-1-up staircases that each terminated after four reversals, converging on the approximate $79 \%$ correct performance level. The initial luminance was the same for both staircases, commencing at $25 \mathrm{~cd} / \mathrm{m}^{2}$ for the high noise case and $8 \mathrm{~cd} / \mathrm{m}^{2}$ for the low noise task. The initial step size was $5.4 \mathrm{~cd} / \mathrm{m}^{2}$ with the step-size halved on the first two reversals 
to reach a final step-size of $1.34 \mathrm{~cd} / \mathrm{m}^{2}$. Individual thresholds were calculated as the mean of the last two reversals of both staircases.

Figure 1C shows the centre-surround contrast stimulus (mean background luminance $172 \mathrm{~cd} / \mathrm{m}^{2}$ ) which was a drifting sine grating (spatial frequency 2 c/deg; drift rate 2 deg/s, randomised drift direction and starting phase) in a circular envelope consisting of a central area (0.67 deg radius) and a surround annulus (outer circumference: 4 deg radius, inner circumference: 0.67 deg radius). The drifting grating was constructed of 8 movie frames, each presented for 66.67 ms ( 2 frames; total stimulus duration $533.33 \mathrm{~ms}$ ). The contrast of the annular stimulus (Figure 1C - left) was fixed at $40 \%$ centrally and 95\% for the annulus (reference stimulus). For the no-surround stimulus (Figure 1C right), the central contrast varied according to two interleaved 1-down-1-up staircases designed to converge on the point of subjective equality. Both staircases commenced with a contrast of $30 \%$ and terminated after six reversals with an initial step size of $8 \%$ contrast, and subsequent steps sizes halved to reach a minimum step size of $2 \%$ contrast. Individual thresholds were calculated as the mean of the last four staircase reversals. Stimulus duration was 400 ms during which observers compared the contrast of both central gratings, before indicating the location of the higher contrast centre by tapping on lower left and right touchscreen regions (3.4 x 3.4 deg of visual angle). The next stimulus was presented 500 ms after a response was registered. 
Stimuli were created using a customised script written in Matlab R2016b (Mathworks, Natick, Massachusetts, United States) run on a personal computer. For each luminance noise and contrast level, ten variants, varying in noise jitter, drift direction and phase, were generated and the image sets uploaded to the PsyPad server. For each stimulus presentation, the PsyPad application randomly presented one of the ten variants to ensure stimulus properties (noise jitter, drift direction, phase) were randomised between trials.

Daily test order was fixed: 1) the migraine survey question; 2) luminance increment detection in high noise; 3) luminance increment detection in low noise and 4) centre-surround contrast discrimination. Participants were instructed to perform the daily test under the same conditions each day, including location, approximately similar time each day and to avoid screen reflections, glare and shadows. All participants were provided with a take-home instruction sheet detailing reminder to maintain consistency in test lighting (see Supplementary Material). 
A

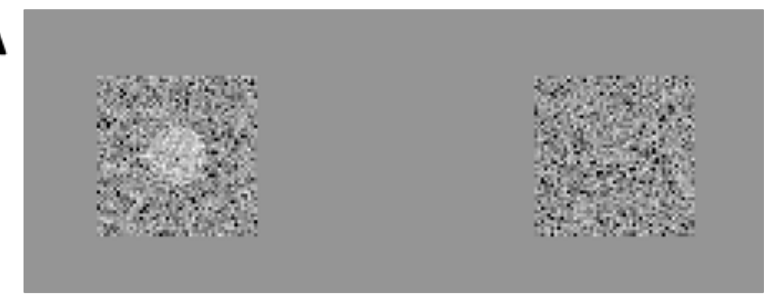

B

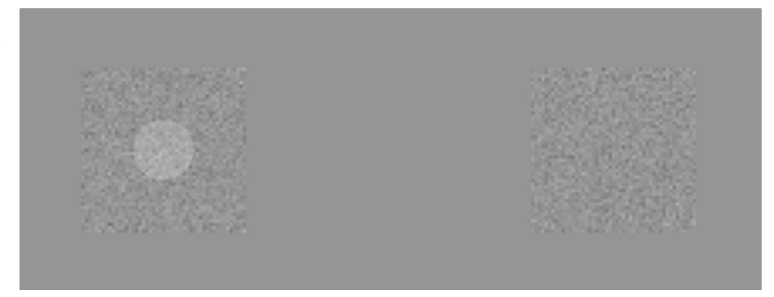

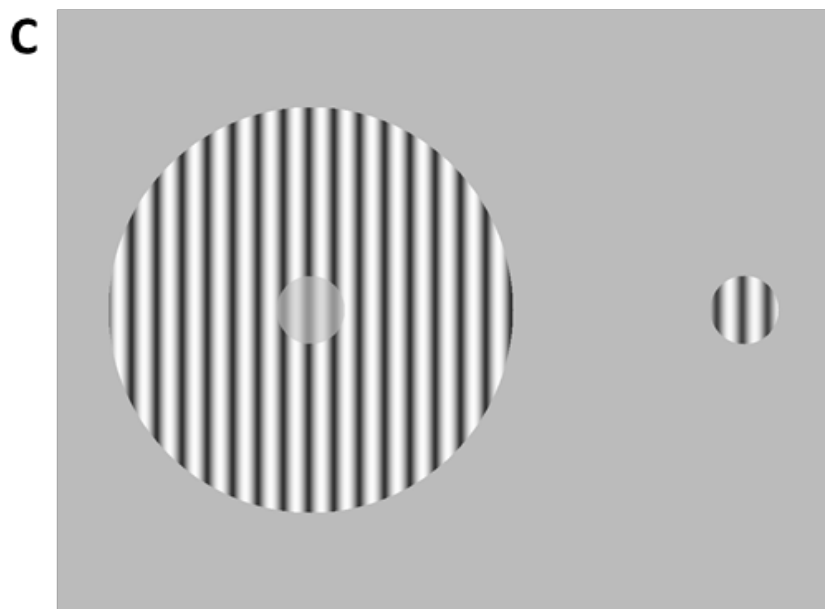

Figure 1: Example of the stimulus used in the luminance increment detection tasks in (A) high and (B) low spatial luminance noise and (C) the centre-surround contrast discrimination task.

\section{Analysis}

Headache free days were defined as more than 2 days before a migraine and more than 2 days after a migraine. This time interval was chosen based on previous work (30-32), and pragmatically because we were able to capture this time window for all our migraine participants. Data for headache free days were pooled within an individual, as were the other timepoints (2 days pre-migraine (2M), 1 day pre-migraine (1M), migraine event (M), 1 day post-migraine (M1), 2 days post-migraine (M2)) if more than one migraine event was captured (12 participants, but most with only one attack with no 
missing data). When attacks lasted for more than one day (but always less than three days to fit the criteria for typical episodic migraine), data within the individual were averaged across all attack days. For controls, data for each individual was averaged across test-days, either for the first and second week of testing separately, or for the entire duration (see below for specific analyses). Missing data was ignored in the averaging process (i.e. if controls did not perform the test on all 14 days; or people with migraine did not perform the tests on all headache free days). Details of non-compliance rates appear in the results.

Statistical analyses were conducted in SPSS Statistics Version 21 (IBM, New York, USA). Group averages were compared using paired t-tests except for when normality test failed where MannWhitney Rank Sum Test was used. To examine changes in luminance increment detection thresholds across migraine time points, a two-way repeated measures analysis of variance (RM-ANOVA factors: noise level x timepoint) was used.

\section{Results}

Compliance rate within the first 14 days of home-monitoring

Control observers were instructed to complete daily testing for 14 days while those with migraine participated until at least 7 days post migraine event. Observers with migraine performed the home testing between 14 to 68 days (mean \pm SD: $33.1 \pm 15.9$ days). Compliance rate was assessed as the 
number of days completed within the first 14 days of home monitoring since there was at most 14 days of data from the controls. Control observers completed between 10 to 14 days (mean \pm SD: 12.8 \pm 1.5 days) while the observers with migraine completed between 6 to 14 days (12.0 \pm 2.3 days). These rates were not different between groups $(\mathrm{t}(32)=1.11, \mathrm{p}=0.27)$.

Exploring for practice effects during the home-monitoring phase

Prior to examining group differences and the changes in perceptual performance within the migraine cycle, we used two approaches to examine if there were any learning/practice effects during the home monitoring phase. Firstly, control data collected from week 1 were compared to week 2. Paired t-tests revealed no significant difference between weeks for any of the tasks (high luminance noise detection thresholds: $\mathrm{t}(15)=0.36, \mathrm{p}=0.73$; low luminance noise detection thresholds: $t(15)=1.02, p=0.32$; contrast matching threshold: $t(15)=-0.05, p=0.96)$. This analysis cannot be conducted for the migraine group due to migraine events occurring within the first two weeks of testing.

We additionally fit regression lines to the individual data for both control and migraine groups for the luminance increment detection task to explore further for learning effects. For the controls, for each individual, all available test points were included in the regression. For the people with 
migraine, only data from non-headache days were included. Within the migraine group 2 out of 18 participants had regression line slopes that were statistically different from zero (improvement). As a proportion, this is not statistically significantly different from 0 out of 18 participants (Chi-square test on proportions, Chi-squared $=0.019, \mathrm{p}=0.89$ ). For the controls, there were 2 of 16 people. These proportions were also not statistically different between the migraine and controls (Chisquared $=0.00, \mathrm{p}=0.99)$.

Secondly, thresholds measured for both tasks in the initial lab session (post instruction and training) (Lab 1), during the home testing phase (Controls: HM; Migraine: headache-free, HF) and in the final lab session (Lab 2) were compared to check for general improvement gained from regular home testing. Luminance increment thresholds were not different across the three time periods (nonsignificant effect of timepoint: $\mathrm{F}(2,64)=2.57, \mathrm{p}=0.08$ ) (Figure 2A, 2B). Perceived contrast thresholds were also not different across the three time periods (non-significant effect of timepoint: $\mathrm{F}(2,64)=1.94, \mathrm{p}=0.15)$ (Figure 2D).

Reduced luminance increment detection thresholds in the migraine group for both high and low noise 
Figure $2 \mathrm{~A}$ and $2 \mathrm{~B}$ shows that luminance increment detection thresholds were higher in the high luminance noise condition than in the low noise condition (main effect of noise level:

$F(1,32)=231.10, p<0.001)$. Observers with migraine had significantly lower luminance increment detection thresholds relative to controls (main effect of group: $F(1,32)=8.49, p<0.01$ ) for both conditions (non-significant noise level $x$ group interaction: $F(1,32)=2.860, p=0.101$ ). While the raw thresholds were reduced, the ratio of the high noise to low noise thresholds were comparable between groups (non-significant effect of group: $\mathrm{F}(1,32)=2.97, \mathrm{p}=0.09$ ) (Figure 2C). This finding is inconsistent with previous reports of greater threshold changes in the presence of high luminance noise in migraine relative to control groups (26).

Reduced centre-surround perceived contrast in the migraineurs

Figure 2D shows group averaged perceived contrast in the centre-surround task. Observers with migraine perceived the central grating at a lower contrast than the controls (more suppression) (main effect of group: $\mathrm{F}(1,32)=9.51, \mathrm{p}<0.01)$. 

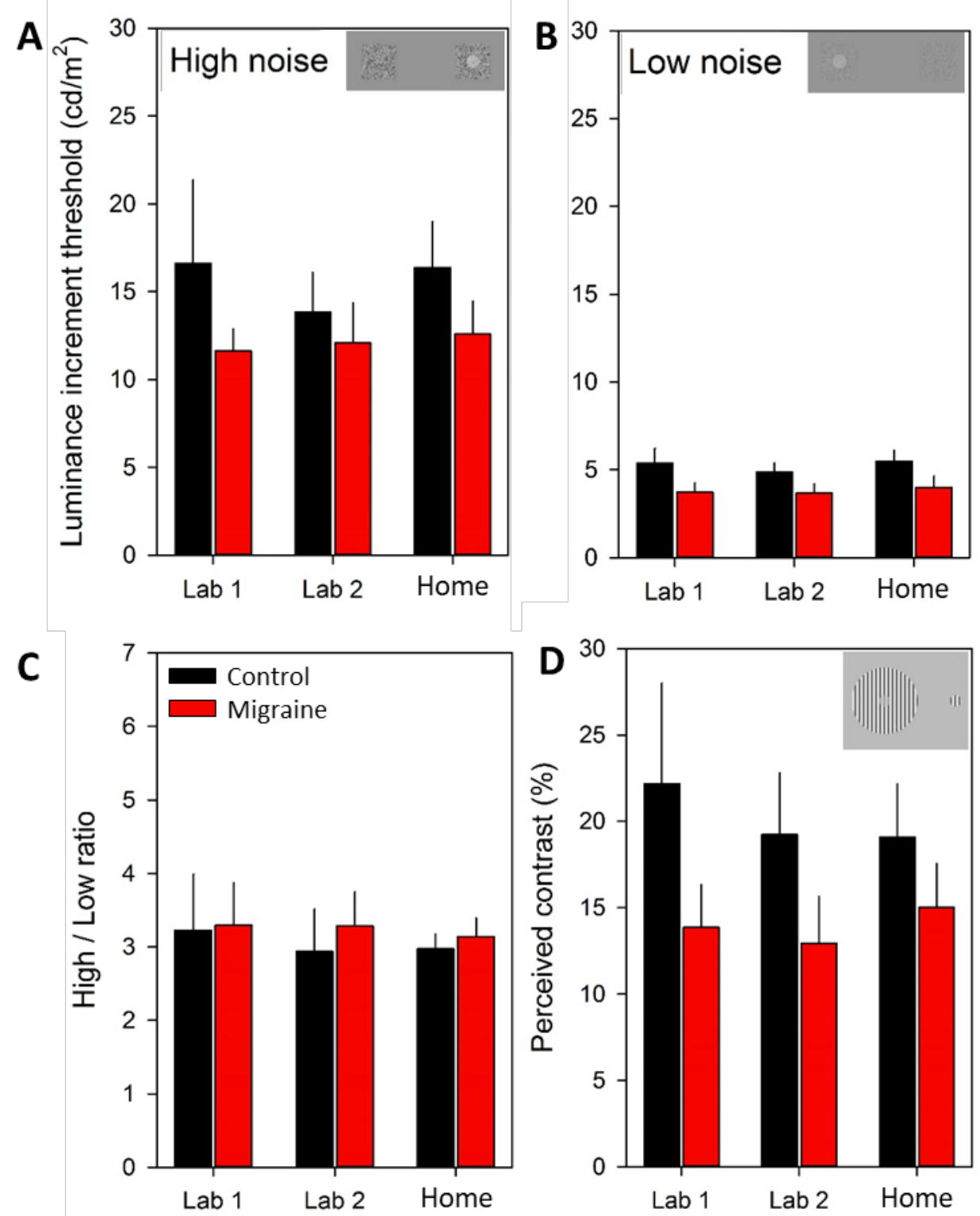

Figure 2: Averaged luminance increment detection thresholds for the control (black) and migraine (red) groups under (A) high luminance noise and (B) low noise conditions at their initial lab session (Lab 1), final lab session post home-monitoring (Lab 2) and home-monitoring test phase (Home: 
note that only the individual headache-free days contributed to this measure for the migraine group). Lower luminance increment detection thresholds were present for the migraine group relative to controls (main effect of group: $\mathrm{F}(1,32)=8.49, \mathrm{p}<0.01$ ) for both high and low noise (non-significant noise level x group interaction: $F(1,32)=2.860, p=0.101$ ) (Panels 2A and 2B). (C) Ratio of the high/low noise thresholds. (D) Group averaged perceived contrast thresholds for the centre-surround contrast suppression task (significant main effect of group: $F(1,32)=9.51, \mathrm{p}<0.01$ ). Error bars represent $95 \%$ confidence intervals of the mean.

No main effect of migraine timepoint on the luminance increment detection thresholds

Figure 3 depicts the average luminance thresholds measured in the migraine group throughout their migraine cycle. For the luminance increment detection task, thresholds were higher for the high noise than low noise condition (main effect of noise level: $F(1,15)=151.69, \mathrm{p}<0.001$ ) but not different across days for either condition (non-significant effect of timepoint: $F(5,75)=1.38, p=0.24$; nonsignificant noise $x$ timepoint interaction: $F(5,75)=0.42, p=0.83)$. The ratio between the threshold in the high and low noise condition was also not altered with days pre- and post-migraine (nonsignificant effect of timepoint: $F(5,75)=0.78, p=0.56)$. After normalising increment detection thresholds to individual thresholds on headache-free days to account for baseline differences, there was still no significant change across days pre- and post-migraine (non-significant effect of timepoint: $\mathrm{F}(4,60)=1.92, \mathrm{p}=0.12)$. 

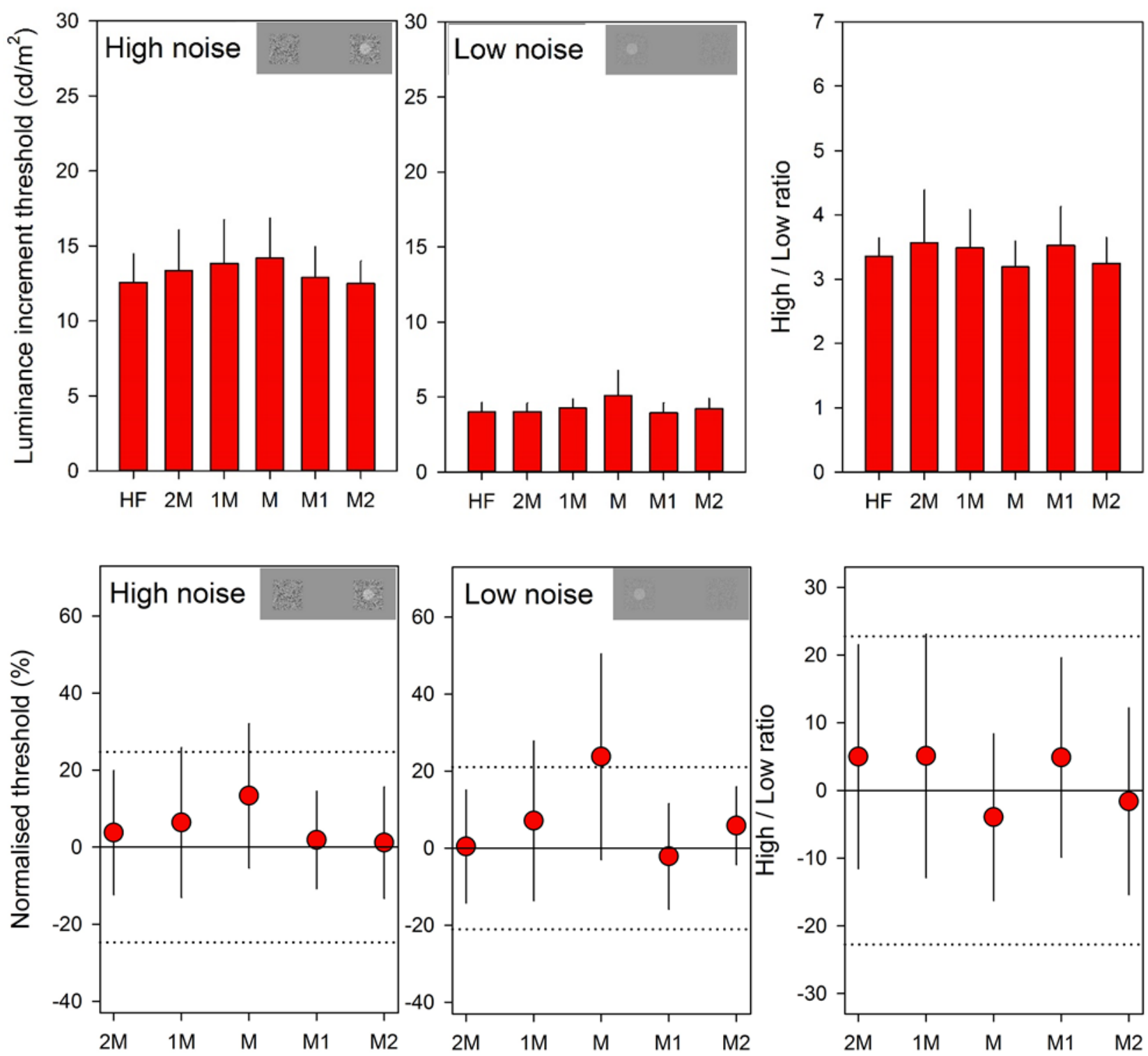

Figure 3: (Top row) Group averaged luminance increment detection thresholds obtained across different timepoints in the migraine cycle (HF - headache-free; $\mathbf{2 M}$ - two days pre-migraine; $\mathbf{1} \mathbf{M}$ one day pre-migraine; $\mathbf{M}$ - attack day(s); $\mathbf{M 1}$ - one day post-migraine; $\mathbf{M} 2$ - two days postmigraine). (Bottom row) Group averaged luminance increment detection thresholds normalised to 20 
individual headache-free days. Dotted line represents the 95\% limits of group variability of the controls. Error bars represent 95\% confidence intervals. There was no significant effect of time-point for any condition.

\section{Reduced centre-surround suppression two days pre-migraine}

For the centre-surround perceived contrast task, performance did alter in proximity to a migraine (main effect of timepoint: $\mathrm{F}(5,75)=3.32, \mathrm{p}<0.01$ ) (Figure 4A). Post-hoc paired t-tests confirmed significantly elevated perceived contrast (reduced surround suppression) 2 days pre-migraine $(\mathrm{t}(17)=-2.23, \mathrm{p}=0.04)$, and a close to significant increase in perceived contrast 1 day pre-migraine $(\mathrm{t}(17)=-2.07, \mathrm{p}=0.06)$. The same group trend holds even after normalising individual perceived contrast thresholds to their own thresholds on headache-free days as a percentage change (main effect of timepoint: $F(4,60)=3.88, p<0.01$ ) (Figure 4B). In order to determine the variance between individuals with respect to the day where the least contrast suppression (highest perceived contrast thresholds) was measured, we report for all individuals the day where they showed the least suppression (Figure 4C), out of all days tested (note, no participants showed lowest suppression during the headache free period). There was some variance between individuals however majority of the observers demonstrated the least suppression pre- or immediately post migraine period. 

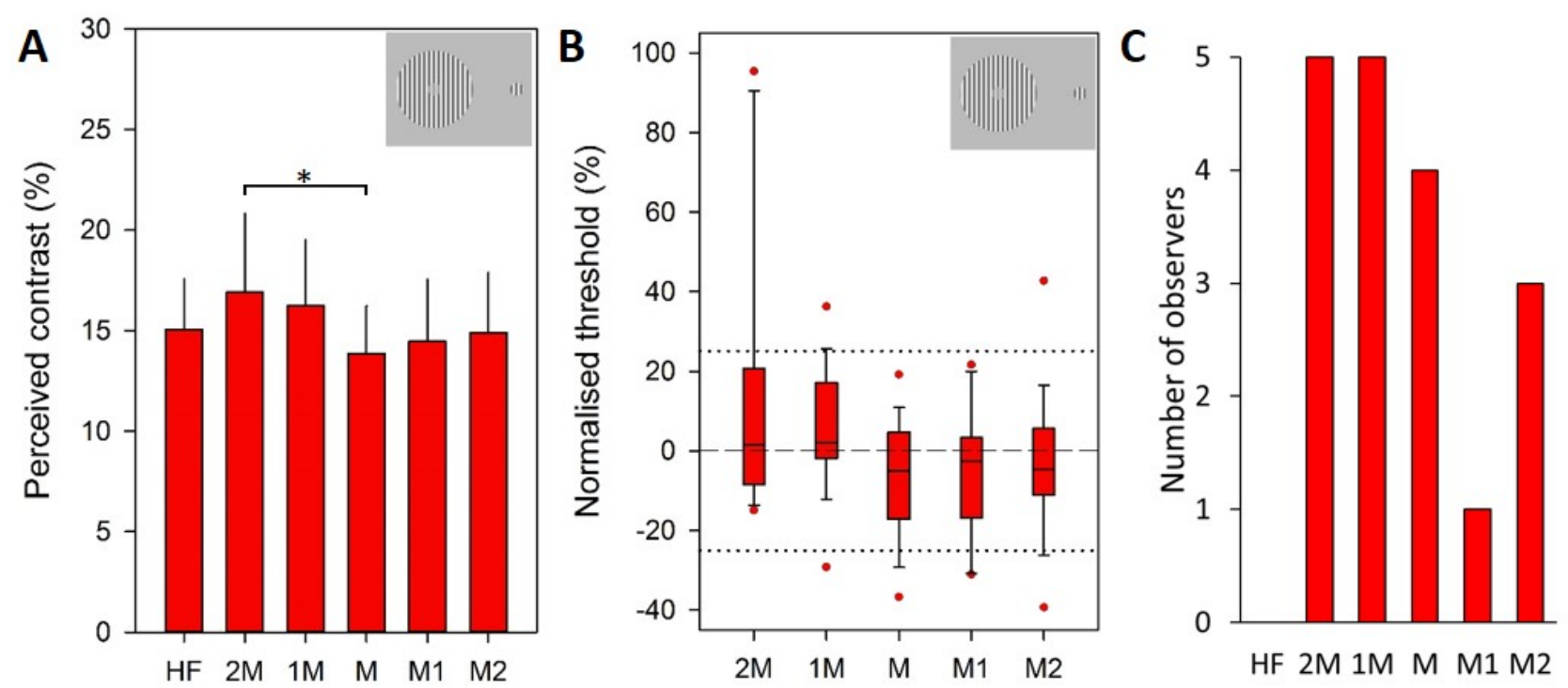

Figure 4: (A) Group averaged perceived contrast thresholds obtained across different timepoints in the migraine cycle (HF - headache-free; $\mathbf{2 M}$ - two days pre-migraine; $\mathbf{1 M}$ - one day pre-migraine; M - attack day(s); M1 - one day post-migraine; M2 - two days post-migraine). Error bars represent 95\% confidence intervals. (B) Group averaged perceived contrast thresholds normalised to individual headache-free days. Dotted line represents the group variability of the controls. Error bars represent $25^{\text {th }}$ and $75^{\text {th }}$ percentiles and symbols represent outliers. (C) Histogram of timepoints where least contrast suppression occurred for each migraine observer. Significantly different performance as a function of timepoint is indicated by the asterisk $(\mathrm{p}<0.05)$. 


\section{Discussion}

Through perceptual testing at home, this study has revealed an on average difference in centresurround contrast suppression in the days pre- and post-migraine (Figure 4A, 4B). Relative to headache-free baseline, the centre surround suppression was weakened (less suppression) at around 48 hours prior to a migraine and was similar to baseline by 24 hours post-migraine. Luminance increment detection thresholds remained unchanged during the migraine event period.

While 14 of 18 (78\%) of our migraine observers demonstrated reduced suppression during migraine build-up, the specific day of least suppression varied (Figure 4C). Our tests were quick, as necessitated to encourage regular testing, so included measurement noise typical of clinical vision testing. For most of our participants, we were only able to capture a single migraine, hence we cannot determine whether contrast suppression alters consistently for an individual with each migraine cycle. Ideally, if this is the case, perceptual testing of contrast suppression could be used as an indicator of likelihood of next migraine. Further studies are required to optimise test protocols and to demonstrate migraine prediction ability, however, our results show some future promise in this regard.

Centre-surround contrast suppression provides an indirect measure of the visual cortical balance in inhibition and excitation $(17,18,33)$. On average, our migraine group perceived the central grating at a lower contrast than the controls (more suppression) on headache-free days (Figure 2D), consistent 23 
with previous reports (19). Based on the daily testing data, we extend that previous work to report here that surround suppression is weakened (higher perceived contrast) at around 48 hours prior to a migraine attack and strengthened to approach their headache-free baseline (lower perceived contrast) by 24 hours post-migraine (Figure 4A, 4B). The precise neuroanatomical mechanisms driving these centre-surround contrast changes cannot be addressed by our study but likely involves an interdependent neurotransmitter cascade. Performance on perceptual centre-surround suppression tasks has been correlated with levels of GABA concentration in human visual cortex as measured by magnetic resonance spectroscopy (21), in addition to influenced by dysregulation of cholinergic mechanisms (34). A plausible interpretation is that the balance between cortical inhibition and excitation swings towards excessive excitation (reduced inhibition and therefore reduced perceptual suppression) in the immediate pre-migraine phase, relatively normalises immediately post-migraine (increasing perceptual contrast suppression), and then tips towards an excessive inhibitory state in the interictal phase (more perceptual contrast suppression than controls). Interestingly, we have previously observed interictal differences in centre-surround suppression of contrast only for stimuli with drifting, rather than static, gratings, implying stronger effects in the areas of the cortex responsible for motion perception (19). We only used drifting gratings here, so cannot comment on replicability, however, comparing performance across the migraine cycle for both static and drifting stimuli in future studies may provide additional insight to the specific visual pathways involved. 
The luminance increment detection task was included because task performance is predicted to change if internal neural noise alters in proximity to a migraine $(25,35)$. Previous work reported elevated thresholds in migraine relative to controls that were interpreted as consistent with a model of increased multiplicative neural noise during the interictal phase using a variant of this stimulus (26). Consequently, we hypothesised that luminance detection thresholds would be elevated in proximity to a migraine event (consistent with increased excitatory neural noise). Our data did not support this hypothesis. Our current methods are not identical to those used previously, in particular, we used a higher mean background luminance than that used by Wagner (2010) and Webster (2012). Indeed, our finding of improved thresholds relative to controls during the headache free phase (Figure 2A, 2B) is consistent with the idea of increased inhibition during this phase. It is worth noting that it is not strictly possible to isolate a mechanism of elevated neural noise relative to other neural activity changes such as gain control regulation via perceptual testing alone, and that mechanistic interpretation of these tasks necessarily requires a degree of speculation. Figure 3 suggests somewhat poorer performance on the day of the migraine, however, this was not statistically significant.

We show in this study that daily home monitoring of vision is feasible, reaching an average compliance rate of around 86\%, comparable to vision home monitoring studies conducted on older adults with macular degeneration $(36,37)$. Some common reasons for failure to comply were "I've forgotten", "I was really busy" and "I was away for a few days". All participants resumed daily 
testing after contact from the researcher. None of the participants had technical issues with the iPad and none complained of the tests being aversive. We did not identify any learning effects resulting in improvement in test performance over time in this study, however it is worth noting that the number of trials for each task were kept abbreviated (to reduce total test time) and no feedback on performance was provided.

This study adds to a large and growing body of work that has used visual perceptual testing, and using other contemporary human neuroscience methods such as brain imaging, electrophysiology and brain stimulation, as non-invasive methods of improving our understanding of migraine pathophysiology. While there is some conjecture in the literature, there are reasonably consistent reports of differences in motion perception, perceptual adaption and measures of gain control between individuals with migraine and controls (for review see: 38,39 and also for review of clinical visual function and electrophysiology see: 25) . Our work provides further evidence for interictal performance differences between migraine events, and further illustrates that the timing of testing within the migraine cycle is important to both specific observations from such testing and their interpretation.

In summary, daily portable testing of vision enabled insight to perceptual performance in the lead up to migraine events, a time point that is typically difficult to capture experimentally. We show that perceptual surround suppression of contrast fluctuates during the migraine cycle, which supports the 26 
utility of this measure as an indirect, non-invasive assay of the balance between cortical inhibition and excitation. Further work is required to refine testing protocols to establish whether perceptual contrast suppression measures can be used to predict migraine events on an individual basis. If successful, the potential for individuals to regulate their own treatment and to prepare for migraine events, will be significantly enhanced. 


\section{Key points}

1. Tablet technology allows daily testing of visual perception throughout the migraine cycle

2. Surround suppression of contrast, an indirect assay of the balance of cortical excitation versus inhibition, is strengthened in the interictal migraine phase.

3. Surround suppression of contrast reduces in strength in the 48 hours prior to a migraine, and normalises 24 hours post migraine.

4. Perceptual tests show some promise as non-invasive accessible tools to identify the premigraine phase.

\section{Research Access}

The visual perceptual tests can be accessed via the PsyPad website:

http://www.psypad.net.au/wiki/Main_Page

\section{References}

1. Blau JN. Migraine: theories of pathogenesis. The Lancet. 1992;339(8803):1202-7.

2. Schulte LH, May A. The migraine generator revisited: continuous scanning of the migraine cycle over 30 days and three spontaneous attacks. Brain. 2016;139(7):1987-93.

3. Schwedt TJ, Chiang C-C, Chong CD, Dodick DW. Functional MRI of migraine. The Lancet Neurology. 2015;14(1):81-91.

4. Hadjikhani N, del Rio MS, Wu O, Schwartz D, Bakker D, Fischl B, et al. Mechanisms of migraine aura revealed by functional MRI in human visual cortex. Proceedings of the National Academy of Sciences. 2001;98(8):4687-92. 
5. Lakhan SE, Avramut M, Tepper SJ. Structural and functional neuroimaging in migraine: insights from 3 decades of research. Headache. 2013;53(1):46-66.

6. Nguyen BN, Vingrys AJ, McKendrick AM. The effect of duration post-migraine on visual electrophysiology and visual field performance in people with migraine. Cephalalgia. 2014;34(1):42-57.

7. Cosentino G, Fierro B, Vigneri S, Talamanca S, Paladino P, Baschi R, et al. Cyclical changes of cortical excitability and metaplasticity in migraine: Evidence from a repetitive transcranial magnetic stimulation study. Pain 2014;155(6):1070-8.

8. Sand T, Zhitniy N, White LR, Stovner LJ. Visual evoked potential latency, amplitude and habituation in migraine: A longitudinal study. Clinical Neurophysiology. 2008;119(5):1020-7.

9. Siniatchkin M, Gerber W-D, Kropp P, Vein A. How the brain anticipates an attack: a study of neurophysiological periodicity in migraine. Functional Neurology. 1999.

10. Siniatchkin M, Sendacki M, Moeller F, Wolff S, Jansen O, Siebner H, et al. Abnormal changes of synaptic excitability in migraine with aura. Cerebral Cortex. 2012;22(10):2207-16.

11. de Tommaso M, Ambrosini A, Brighina F, Coppola G, Perrotta A, Pierelli F, et al. Altered processing of sensory stimuli in patients with migraine. Nature Reviews Neurology. 2014;10(3):144-55.

12. Maniyar FH, Sprenger T, Monteith T, Schankin C, Goadsby PJ. Brain activations in the premonitory phase of nitroglycerin-triggered migraine attacks. Brain. 2013;137(1):232-41.

13. Chubb C, Sperling G, Solomon JA. Texture interactions determine perceived contrast. Proceedings of the National Academy of Sciences. 1989;86(23):9631-5.

14. Yu C, Klein SA, Levi DM. Surround modulation of perceived contrast and the role of brightness induction. Journal of Vision. 2001;1(1):3-.

15. Xing J, Heeger DJ. Measurement and modeling of center-surround suppression and enhancement. Vision Research. 2001;41(5):571-83.

16. Cannon MW, Fullenkamp SC. Spatial interactions in apparent contrast: inhibitory effects among grating patterns of different spatial frequencies, spatial positions and orientations. Vision Research. 1991;31(11):1985-98.

17. Ozeki H, Sadakane O, Akasaki T, Naito T, Shimegi S, Sato H. Relationship between excitation and inhibition underlying size tuning and contextual response modulation in the cat primary visual cortex. Journal of Neuroscience. 2004;24(6):1428-38.

18. Schwabe L, Obermayer K, Angelucci A, Bressloff PC. The role of feedback in shaping the extra-classical receptive field of cortical neurons: a recurrent network model. Journal of Neuroscience. 2006;26(36):9117-29. 
19. Battista J, Badcock DR, McKendrick AM. Migraine increases centre-surround suppression for drifting visual stimuli. PLoS One. 2011;6(4):e18211.

20. Tibber MS, Anderson EJ, Bobin T, Antonova E, Seabright A, Wright B, et al. Visual Surround Suppression in Schizophrenia. Frontiers in Psychology. 2013;4:88.

21. Yoon JH, Maddock RJ, Rokem A, Silver MA, Minzenberg MJ, Ragland JD, et al. GABA concentration is reduced in visual cortex in schizophrenia and correlates with orientationspecific surround suppression. Journal of Neuroscience. 2010;30(10):3777-81.

22. Karas R, McKendrick AM. Age related changes to perceptual surround suppression of moving stimuli. Seeing and Perceiving. 2012;25(5):409-24.

23. Yazdani P, Read JCA, Whittaker RG, Trevelyan AJ. Assessment of epilepsy using noninvasive visual psychophysics tests of surround suppression. Physiological Reports. 2017;5(5).

24. McKendrick AM, Badcock DR. Decreased visual field sensitivity measured 1 day, then 1 week, after migraine. Investigative Ophthalmology and Visual Science. 2004;45:1061-70.

25. Nguyen BN, Lek JJ, Vingrys AJ, McKendrick AM. Clinical impact of migraine for the management of glaucoma patients. Progress in Retinal and Eye Research. 2016;51:107-24.

24. Webster KE, Dickinson JE, Battista J, McKendrick AM, Badcock DR. Evidence for increased internal noise in migraineurs for contrast and shape processing. Cephalalgia. 2012;32(2):12539.

25. Wagner D, Manahilov V, Loffler G, Gordon GE, Dutton GN. Visual noise selectively degrades vision in migraine. Investigative ophthalmology \& visual science. 2010;51(4):2294-9.

26. Headache Classification Committee of the International Headache Society. The international classification of headache disorders, (beta version). Cephalalgia. 2013;33(9):629-808.

27. Turpin A, Lawson DJ, McKendrick AM. PsyPad: A platform for visual psychophysics on the iPad. Journal of vision. 2014;14(3):16-.

28. Bjørk M, Sand T. Quantitative EEG power and asymmetry increase $36 \mathrm{~h}$ before a migraine attack. Cephalalgia. 2008;28(9):960-8.

29. Bjørk MH, Stovner LJ, Engstrøm M, Stjern M, Hagen K, Sand T. Interictal quantitative EEG in migraine: a blinded controlled study. The Journal of Headache and Pain. 2009;10(5):331-9.

30. Cao Z-H, Ko L-W, Lai K-L, Huang S-B, Wang S-J, Lin C-T, editors. Classification of migraine stages based on resting-state EEG power. Neural Networks (IJCNN), 2015 International Joint Conference on; 2015: IEEE. 
31. Angelucci A, Bressloff PC. Contribution of feedforward, lateral and feedback connections to the classical receptive field center and extra-classical receptive field surround of primate V1 neurons. Progress in Brain Research. 2006;154:93-120.

32. Kosovicheva AA, Sheremata SL, Rokem A, Landau AN, Silver MA. Cholinergic enhancement reduces orientation-specific surround suppression but not visual crowding. Frontiers in Behavioral Neuroscience. 2012;6:61.

33. Lu Z-L, Dosher BA. Characterizing observers using external noise and observer models: assessing internal representations with external noise. Psychological Review. 2008;115(1):44.

34. Chew EY, Clemons TE, Agron E, Martin DF, Bressler SB, Gensler G, et al. The Lack of Progression of Age-Related Macular Degeneration following Cataract Surgery in the AgeRelated Eye Disease Study 2 (AREDS2). Investigative Ophthalmology \& Visual Science. 2015;56(7):2572-.

35. Thomas M, Wolfson Y, Zayit-Soudry S, Bressler SB, Bressler NM. Qualifying to use a home monitoring device for detection of neovascular age-related macular degeneration. JAMA Ophthalmology. 2015;133(12):1425-30.

36. O'Hare L, Hibbard P. Visual processing in migraine. Cephalalgia. 2016;36:1057-76.

37. Shepherd AJ, Joly-Mascheroni RM. Visual motion processing in migraine: enhanced motion after-effects are related to display contrast, visual symptoms, visual triggers and attack frequency. Cephalalgia 2017. 2017;37:315-26. 